\title{
Distributed Algorithms for Fully Personalized PageRank on Large Graphs
}

\author{
Wenqing Lin \\ Interactive Entertainment Group, Tencent Inc. \\ edwlin@tencent.com
}

\begin{abstract}
Personalized PageRank (PPR) has enormous applications, such as link prediction and recommendation systems for social networks, which often require the fully PPR to be known. Besides, most of real-life graphs are edge-weighted, e.g., the interaction between users on the Facebook network. However, it is computationally difficult to compute the fully PPR, especially on large graphs, not to mention that most existing approaches do not consider the weights of edges. In particular, the existing approach cannot handle graphs with billion edges on a moderate-size cluster. To address this problem, this paper presents a novel study on the computation of fully edge-weighted PPR on large graphs using the distributed computing framework. Specifically, we employ the Monte Carlo approximation that performs a large number of random walks from each node of the graph, and exploits the parallel pipeline framework to reduce the overall running time of the fully PPR. Based on that, we develop several optimization techniques which (i) alleviate the issue of large nodes that could explode the memory space, (ii) pre-compute short walks for small nodes that largely speedup the computation of random walks, and (iii) optimize the amount of random walks to compute in each pipeline that significantly reduces the overhead. With extensive experiments on a variety of real-life graph datasets, we demonstrate that our solution is several orders of magnitude faster than the state-of-the-arts, and meanwhile, largely outperforms the baseline algorithms in terms of accuracy.
\end{abstract}

\section{CCS CONCEPTS}

- Information systems $\rightarrow$ Social networks; Personalization; • Computing methodologies $\rightarrow$ MapReduce algorithms.

\section{KEYWORDS}

Distributed Algorithm; Graph; Personalized PageRank

\section{ACM Reference Format:}

Wenqing Lin. 2019. Distributed Algorithms for Fully Personalized PageRank on Large Graphs. In Proceedings of the 2019 World Wide Web Conference (WWW'19), May 13-17, 2019, San Francisco, CA, USA. ACM, New York, NY, USA, 11 pages. https://doi.org/10.1145/3308558.3313555

\section{INTRODUCTION}

Given a graph $G=(V, E, w)$, and two nodes $u, v \in V$, the personalized PageRank (PPR) of $v$ with respect to $u$, denoted by $\pi(u, v)$,

This paper is published under the Creative Commons Attribution 4.0 International (CC-BY 4.0) license. Authors reserve their rights to disseminate the work on their personal and corporate Web sites with the appropriate attribution.

WWW'19, May 13-17, 2019, San Francisco, CA, USA

() 2019 IW3C2 (International World Wide Web Conference Committee), published under Creative Commons CC-BY 4.0 License.

ACM ISBN 978-1-4503-6674-8/19/05.

https://doi.org/10.1145/3308558.3313555 is defined as the probability of a random walk starting from $u$ and ending at $v$. Compared to PageRank (PR), which is a global metric of nodes, PPR depicts the asymmetric perspective of each node to all the other nodes, i.e., $\pi(u, v)$ is not necessarily equal to $\pi(v, u)$. There are a plethora of applications that utilize PPR to measure the proximity of two nodes in the graph, such as recommendation system [5, 19, 21] and natural language processing [1]. In these applications, the fully PPR, i.e., the PPRs of all pairs of nodes, are often required. In other words, for all node $v$ in the graph, the PPR of all the other nodes $u$ with respect to $v$ should be known.

However, most of previous work $[9,11,16]$ focus on the computation of single-pair or single-source PPR, rendering them unsuitable for solving the problem of fully PPR. For example, FORA [27] is the recent algorithm for single-source PPR, and needs 103 seconds to answer a single-source query on a Twitter graph of 41.7 millions of nodes, which would require about 137 years to compute the single-source PPRs for all nodes. In addition, it is highly difficult to compute the fully PPR, especially on large graphs. In particular, the probability of a random walk is usually computed by the Power Iteration method [8] or approximated by the Monte Carlo method [7], which might require a large number of iterations of computation to obtain a converged results. Besides, most of existing methods are tailored for the setting of a single machine [7], which renders them unable to handle graphs that cannot fit in memory, not to mention that the space complexity of the fully PPR could be $O\left(n^{2}\right)$ where $n$ is the number of nodes in the graph. What's more, most real-world graphs are weighted, which can be directly generated from the interactions between nodes. For instance, the weight of the edge from a user $u$ to another user $v$ in the Facebook network could be the number of comments from $u$ to the posts of $v$. Nevertheless, most existing methods do not consider the weights on graph, making their algorithms unsuitable.

To address these issues, we devise a distributed share-nothing solution that employs the Monte Carlo approximation to perform a large number of random walks from all nodes of the graph in parallel. As such, for each node $u \in V$, we maintain only the PPR $\pi(u, v)$ where $v \in V$ is the node incurred in at least one random walk. Besides, we exploit the parallel pipeline framework [13] where (i) a subset of random walks is computed simultaneously in a pipeline and (ii) the new pipeline can remedy the deficiency of the old pipeline, as it is rare to generate long random walks due to the termination probability. Hence, it reduces the number of iterations of computation that significantly accelerates the process.

In order to optimize the above solution, we first address the issue of large nodes that would lead to the skewness of data. Specifically, we recursively divide each large node into small ones, which are then organized as the alias tree in a hierarchical manner. In addition, 
to address the issue of under-utilization of small nodes, we precompute for each small node a set of short walks to facilitate the acceleration of random walk generation on small nodes. Finally, since it would significantly degrade the performance of a pipeline which deals with very few or too many random walks at a time, we develop a method that optimizes the number of random walks in each pipeline so as to reduce the overhead of pipelining.

In summary, our contributions are the followings.

- We devise the distributed algorithms based on the parallel pipeline framework that fully utilizes the parallelism to efficiently generate a large number of random walks simultaneously for all nodes of the graph.

- We design the hierarchical sampling algorithm that avoids the skewness on large nodes and also allows the algorithm to select a node from the set of neighbors following the distribution of weights on edges in constant time.

- We develop an efficient algorithm to deal with small nodes by pre-computing short walks so that some random walks can be stopped earlier, which saves lots of costs.

- We devise an effective method to optimize the number of random walks in each pipeline which largely increases the throughput of the algorithm that greatly improves its performance on large graphs.

- In the extensive experiments, we demonstrate that our solution outperforms the state-of-the-art solutions by up to $21 \%$ on accuracy and by up to 2 orders of magnitude on the running time.

Paper organization. Section 2 illustrates the definitions and notations used in the paper. An overview of our solution is depicted in Section 3. After that, the details of the algorithm are explained in Section 4. Section 5 demonstrates the superior performance of our algorithm compared with several baseline methods over many graphs. Section 6 discusses the related work of this paper. Finally, we conclude the paper in Section 7.

\section{PRELIMINARIES}

In this section, we present the concepts and frequently used notations in this paper, as listed in Table 1.

\subsection{Personalized PageRank}

Consider an edge-weighted graph $G=(V, E, w)$, where $V$ is the set of nodes, $E$ is the set of edges, and $w$ is the weighting function that maps each edge $e \in E$ to a positive number $w(e)$, i.e., $w(e)>0$. For each node $s \in V$, we say that $t$ is an out-neighbor (resp. in neighbor) of $s$, if there exists an edge $\langle s, t\rangle$ (resp. $\langle t, s\rangle)$ such that $\langle s, t\rangle \in E($ resp. $\langle t, s\rangle \in E)$. Denote $N_{\text {out }}(s)\left(\right.$ resp. $\left.N_{\text {in }}(s)\right)$ as the set of out-neighbors (resp. in-neighbors) of $s$.

Given an out-neighbor $t \in N_{\text {out }}(s)$ of a node $s \in V$, we refer to the routing probability of $t$ with respect to $s$ as $r(s, t)=$ $w(\langle s, t\rangle) / \sum_{v \in N_{\text {out }}(s)} w(\langle s, v\rangle)$. As such, we have $r(s, t) \in(0,1]$.

Given a graph $G=(V, E, w)$, a walk or path $P$ of $G$ is a sequence of nodes, denoted by $\left\langle v_{1}, v_{2}, \cdots, v_{k}\right\rangle$, such that (i) $v_{i} \in V$ for each $i \in[1, k]$ and (ii) $\left\langle v_{i}, v_{i+1}\right\rangle \in E$ for each $i \in[1, k-1]$. The length of $P$, denoted by $\ell(P)$, is the number of edges in $P$, i.e., $\ell(P)=k-1$.
A random walk $P_{r}=\left\langle v_{1}, v_{2}, \cdots, v_{k}\right\rangle$ of $G$ is a path that is generated in a random manner following the distribution of routing probability. Specifically, for each node $v_{i}$ where $i \in[1, k-1]$, we have $v_{i+1} \in N_{\text {out }}\left(v_{i}\right)$ and $v_{i+1}$ is chosen with the routing probability $r\left(v_{i}, v_{i+1}\right)$. Furthermore, at each step of the random walk $P_{r}$, there is a termination probability $\alpha \in(0,1)$, which is a user-defined number, that determines whether the path will be terminated or not. In other words, after choosing $v_{i+1}, P_{r}$ will stop at $v_{i+1}$ with the termination probability $\alpha$. Hence, the expected length of a random walk is $1 / \alpha$. In case that $N_{\text {out }}\left(v_{i}\right)$ is empty, following the previous work [27], we restart $P_{r}$ from its head, i.e., $v_{1}$.

Example 2.1. Figure 1 shows a graph $G$ which has 8 nodes and 12 weighted edges. The weights of edges incident to each node are normalized such that the routing probability of each edge equals its weight. Consider node $v_{0}$ and $\alpha=0.5$. We have $N_{\text {out }}\left(v_{0}\right)=$ $\left\{v_{1}, v_{2}, v_{3}, v_{4}, v_{5}, v_{6}\right\}$ and $N_{i n}\left(v_{0}\right)=\left\{v_{5}\right\}$. The path $\left\langle v_{0}, v_{3}, v_{4}\right\rangle$ is walk starting from $v_{0}$ and ending at $v_{4}$, which can be generated by (i) first selecting $v_{3}$ from $N_{o u t}\left(v_{0}\right)$ with the probability 0.15 , (ii) and then with the probability of $(1-\alpha)=0.5$ continuing to select $v_{4}$ from $N_{\text {out }}\left(v_{3}\right)$ with the probability 1 , (iii) finally stopping at $v_{4}$ with the termination probability $\alpha=0.5$. Besides, there exist the other paths that start from $v_{0}$ and end at $v_{4}$, such as $\left\langle v_{0}, v_{4}\right\rangle$ and $\left\langle v_{0}, v_{2}, v_{3}, v_{4}\right\rangle$.

Given a graph $G=(V, E, w)$ and two nodes $u, v \in V$, the personalized PageRank (PPR) of $v$ with respect to $u$, denoted by $\pi(u, v)$, is the probability of a random walk which starts from $u$ and ends at $v$.

To address this problem, there are roughly two kinds of solutions: One is the matrix based solution that utilizes the adjacency matrix of the graph and the matrix of fully PPR, the other one is based on the Monte Carlo (MC) method that estimates the probability by simulating a large number of random walks on the graph. However, the matrix-based solution incurs a space complexity of $O\left(n^{2}\right)$ where $n$ is the number of nodes in the graph, which is extremely huge, especially for large graph. As such, we adopt the MC based solution in our proposed approach.

Specifically, for each node $s \in V$, the MC method generates a number $\omega$ of random walks starting from $s$ to estimate $\pi(s, t)$ for every other node $t \in V$. If there are $\omega^{\prime}$ random walks terminating at $t$, then we have an unbiased estimate of $\pi(s, t)$ as $\hat{\pi}(s, t)=\omega^{\prime} / \omega$. It is easy to see that a larger $\omega$ leads to a more accurate estimate of $\pi(s, t)$, and also a much more expensive computational cost. To strike a good trade-off, we follow the theorem in the work [7] by setting $\omega=\Omega\left(\frac{\log \left(1 / p_{f}\right)}{\epsilon^{2} \delta}\right)$ to obtain an $\epsilon$-approximate PPR for any $\pi(s, t) \geq \delta$ with a probability $1-p_{f}$, where $s, t \in V$, which is formally defined as follows.

Definition 2.2 (Fully Approximate PPR (FAPPR)). Given an edgeweighted graph $G=(V, E, w)$, a threshold $\delta$, an error bound $\epsilon$, and a failure probability $p_{f}$, the fully approximate PPR returns an estimated PPR $\hat{\pi}(s, t)$ for all pairs $(s, t)$ of nodes where $s, t \in V$, such that for all $\pi(s, t) \geq \delta$,

$$
|\pi(s, t)-\hat{\pi}(s, t)| \leq \epsilon \cdot \pi(s, t)
$$

holds with a probability at least $1-p_{f}$. 


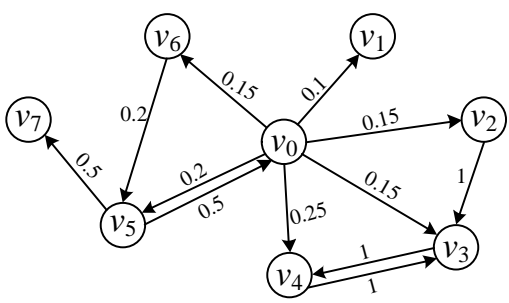

Figure 1: A toy graph with 8 nodes and 12 weighed edges.

Goal. In the present work, we are to devise efficient distributed algorithms for the computation of fully approximate PPR on large graphs.

\subsection{The Alias Method}

One of the key operations in random walk is to select one node $t$ from $s$ 's out-neighbors, where $s \in V$, according to the routing probability. In other words, given a set $S$ of elements, as well as the routing probability $r(e)$ for each $e \in S$, where $\sum_{e \in S} r(e)=1$, we are to select an element $e$ from $S$ with the probability $r(e)$.

In a naive approach, similar to [4], we can identify an element $e$ from $S$ which has the largest $z^{1 / r(e)}$, where $z \in[0,1]$ is a random number. Consequently, this approach needs to inspect each element in $S$ on the fly, whose time complexity is $O(|S|)$. However, it might lead to the workload imbalance in the distributed computing algorithm on the graph, as the sets of out-neighbors could be of different sizes.

We present the alias method [25], which pre-computes a switch probability $p(e) \in[0,1]$ and an alias $a(e) \in S$ for each element $e \in S$. As such, an element $e$ can be selected from $S$ with constant time complexity by exploiting $p(e)$ and $a(e)$.

Algorithm 1 illustrates the pre-computing step of the alias method that takes as input the routing probability $r$ and the set $S$ of elements, i.e., edges. Initially, for each edge $e \in S$, its alias $a(e)$ is set as $e$, and its switch probability $p(e)$ is computed as $|S| \cdot r(e)$ (Line 1$)$. Thus, we have $\sum_{e \in S} p(e)=|S|$ at the beginning of the algorithm. Denote $S^{l}\left(\right.$ resp. $S^{s}$ ) as the set of elements $e$ where $p(e)>1$ (resp. $p(e)<1)\left(\right.$ Line 2). Then, we choose an element $x$ from $S^{s}$ and an element $y$ from $S^{l}$ respectively. After that, we update the alias of $x$ as $a(x)=y$, and decrease the probability of $y$ by $(1-p(x))$, referred to as $p^{\prime}(y)=p(y)-(1-p(x))$. As $x$ is processed, we remove $x$ from $S_{s}$. Besides, If $p^{\prime}(y) \leq 1$, then we remove $y$ from $S^{l}$. Furthermore, if $p^{\prime}(y)<1$, then we add $y$ into $S^{s}$. We iteratively process the elements in $S^{l}$, until $S^{l}$ is empty (Line 3-9). Finally, we have $p(e)$ and $a(e)$ for all $e \in S$ as the results. Since each element in $S$ is inspected only once, the time complexity of this pre-computing step is $O(|S|)$.

Example 2.3. Consider the set $S_{1}=\left\{e_{1}, e_{2}, e_{3}\right\}$ in Fig. 3. Consider the edges $e$ in $S_{1}$. The routing probability of $e_{1}$ is computed as $r\left(e_{1}\right)=w\left(e_{1}\right) / \sum_{e \in S_{1}} w(e)=0.25$. Similarly, we have $r\left(e_{2}\right)=r\left(e_{3}\right)=0.375$. At the beginning, the switch probability of $e_{1}$ is computed as $p\left(e_{1}\right)=\left|S_{1}\right| \cdot r\left(e_{1}\right) / \sum_{e \in S_{1}} r(e)=0.75$. Similarly, we have $p\left(e_{2}\right)=p\left(e_{3}\right)=1.125$. Besides, we have $a\left(e_{i}\right)=e_{i}$ where $1 \leq i \leq 3$. As such, we construct $S_{1}^{l}=\left\{e_{2}, e_{3}\right\}$ since $p\left(e_{2}\right)>1$ and $p\left(e_{3}\right)>1$, and $S_{1}^{S}=\left\{e_{1}\right\}$ since $p\left(e_{1}\right)<1$. Assume that $e_{1} \in S_{1}^{s}$ and $e_{2} \in S_{1}^{l}$ are selected. Then, we update $a\left(e_{1}\right)$ as $e_{2}$, remove $e_{1}$

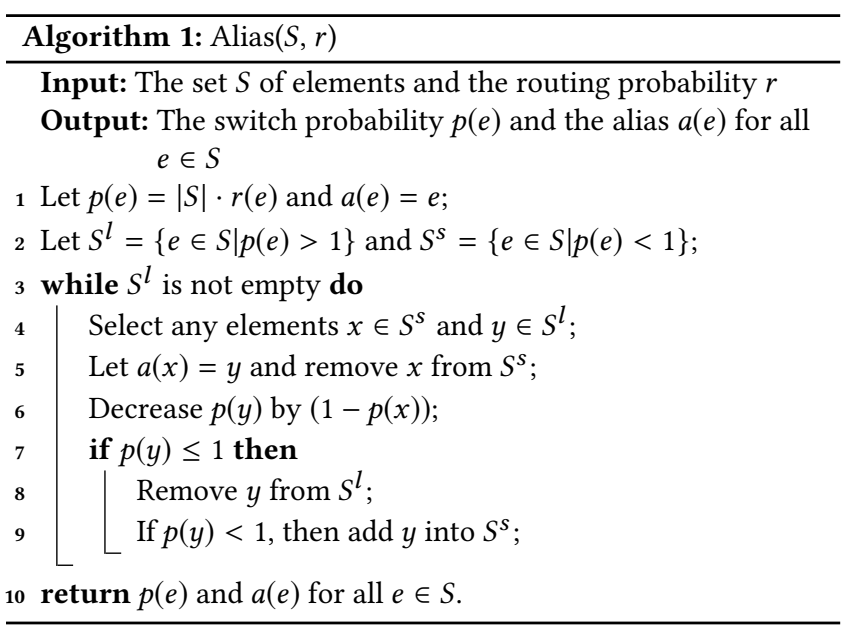

from $S_{1}^{s}$, and decrease $p\left(e_{2}\right)$ by $\left(1-p\left(e_{1}\right)\right)$, resulting in $p\left(e_{2}\right)=0.875$. Since $p\left(e_{2}\right)<1$, we remove $e_{2}$ from $S_{1}^{l}$ and add $e_{2}$ into $S_{1}^{s}$. Then, we have $S_{1}^{s}=\left\{e_{2}\right\}$ and $S_{1}^{l}=\left\{e_{3}\right\}$. In the same way, we can compute that $a\left(e_{2}\right)=e_{3}$ and $p\left(e_{3}\right)=1$, which forms the aliases and switch probabilities of $S_{1}$, as shown in Fig. 3.

To select an element $e$ from $S$ with the switch probability $p(e)$ and the alias $a(e)$, we first identify an element $e^{\prime}$ with the probability $1 /|S|$. Then, a probability threshold $p_{t} \in[0,1]$ is generated by random. If $p_{t} \leq p\left(e^{\prime}\right)$, then we have $e=e^{\prime}$; Otherwise, let $e$ be the alias of $e^{\prime}$, i.e., $e=a\left(e^{\prime}\right)$. Therefore, the probability of selecting $e$

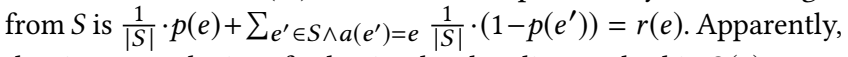
the time complexity of selecting by the alias method is $O(1)$.

\subsection{Distributed Computing}

However, it is impractical to compute FAPPR on a single machine, since (i) the graph could be massive that cannot fit in the memory of a single machine, and (ii) there could be an extremely large amount of computational cost due to lots of graph traversals, especially when the graph is sufficiently large, which also results in a large number of random walks (see Section 2.1).

As such, we utilize the distributed computing to generate the large number of random walks for all nodes of the graph among massive machines in parallel. In particular, we adopt Spark [30], whose fundamental computing unit is MapReduce [3,23]. Note that, it is easy to extend to the other parallel computing framework, such as Pregel [20]. In a MapReduce job, there are mainly two consequent phases: The first one is the Map phase that takes as input the data and emits key-value pairs by the map function; After that, it is the Reduce phase that aggregates the key-value pairs by the key and then applies the reduce function on the aggregated data, which outputs the new key-value pairs.

To facilitate the processing of graphs on Spark, we store the graph as key-value pairs, such that, for each node $v \in V$, we a key-value pair $\left\langle v, N_{\text {out }}(v)\right\rangle$ where the key is $v$ and the value is the set of $v$ 's out-neighbors, i.e., $N_{\text {out }}(v)$. Denote the set of key-value pairs of $G$ as $K V(G)=\left\{\left\langle v, N_{\text {out }}(v)\right\rangle \mid v \in V\right\}$. Besides, to present a path $P=\left\langle v_{h}, \cdots, v_{t}\right\rangle$, we use a key-value pair $\left\langle v_{t}, v_{h}\right\rangle$ consisting of the tail $v_{t}$ of $P$ as the key and the head $v_{h}$ of $P$ as the value. This 
Table 1: Frequently used notations.

\begin{tabular}{|l|l|}
\hline Notation & Description \\
\hline$G=(V, E, w)$ & $\begin{array}{l}\text { an edge-weighted and directed graph, where } V \text { is the set } \\
\text { of nodes, } E \text { is the set of edges, and } w \text { is the weighting } \\
\text { function that maps each edge in } E \text { to a positive number. }\end{array}$ \\
\hline$r$ & the routing probability of an edge (see Section 2.1). \\
\hline$\omega$ & $\begin{array}{l}\text { the number of random walks for each node (see Defini- } \\
\text { tion 2.2). }\end{array}$ \\
\hline$\gamma$ & $\begin{array}{l}\text { the number of random walks in each pipeline (see Defi- } \\
\text { nition 2.2). }\end{array}$ \\
\hline$\alpha$ & $\begin{array}{l}\text { the termination probability of a random walk (see Defi- } \\
\text { nition 2.2). }\end{array}$ \\
\hline$\epsilon$ & the relative accuracy guarantee (see Definition 2.2). \\
\hline$\delta$ & the PPR value threshold (see Definition 2.2). \\
\hline$p$ & the switch probability of an edge in the alias tree. \\
\hline$a$ & the alias of an edge in the alias tree. \\
\hline$d$ & the degree threshold in Algorithm 3 and Algorithm 4. \\
\hline
\end{tabular}

is because (i) only the head and tail of a random walk can contribute to the calculation of PPR, and (ii) the extension of a path happens at its tail.

Based on that, given a set $\mathcal{P}$ of paths, we can make a further move or step for all paths $P \in \mathcal{P}$ by one round of computation on Spark that utilizes a MapReduce job: First, the Map phase takes as input both the key-value pairs $\left\langle v_{h}, v_{t}\right\rangle$ of all paths $P \in \mathcal{P}$ and the key-value pairs $\left\langle v, N_{\text {out }}(v)\right\rangle \in K V(G)$ of the graph $G$, and emits the key-value pairs as what they are; Then, in the Reduce phase, we aggregates the key-value pairs by the key, resulting in that each aggregated data has one $\left\langle v_{h}, N_{o u t}\left(v_{h}\right)\right\rangle$ and several other key-value pairs $\left\langle v_{h}, u\right\rangle$ that represent the paths starting from $u$ and currently visiting $v_{h}$. For each $\left\langle v_{h}, u\right\rangle$, we select a node $v_{h}^{\prime}$ from $N_{\text {out }}\left(v_{h}\right)$ with the routing probability $r\left(v_{h}, v_{h}^{\prime}\right)$, and output a new key-value pair $\left\langle v_{h}^{\prime}, u\right\rangle$, which represents the extended path of $\left\langle v_{h}, u\right\rangle$.

Note that, one can implement the above algorithm by exploiting the join operation on Spark. As such, it can be optimized by a careful designed data partitioning scheme [31], which can avoid the cost of transferring between machines the set $K V(G)$ of key-value pairs of $G$.

\section{SOLUTION OVERVIEW}

In this section, we present an overview of our distributed algorithm for the computation of the fully approximate edge-weighted PPR (see Definition 2.2). As the major cost of FAPPR resides in the computation of random walks, in the sequel, we will concentrate our discussions on the algorithms for generating random walks.

Given a graph $G=(V, E, w)$, to generate $\omega$ random walks for each node $v \in V$ in the distributed computing framework, one straightforward solution could run in $\omega$ iterations, each of which computes one random walk for each node. In particular, one random walk $P$ ending with the node $v$ can be extended by one more step in one round of the distributed computing, where we append $P$ with one node randomly chosen from $N(v)$. However, as the expected length of a random walk is $1 / \alpha$, this approach results in $\omega / \alpha$ number of rounds of computation, which is highly expensive. Alternatively, one might propose to generate all $\omega$ random walks for each node $v \in V$ using one iteration with only $1 / \alpha$ rounds, where $\omega$ number

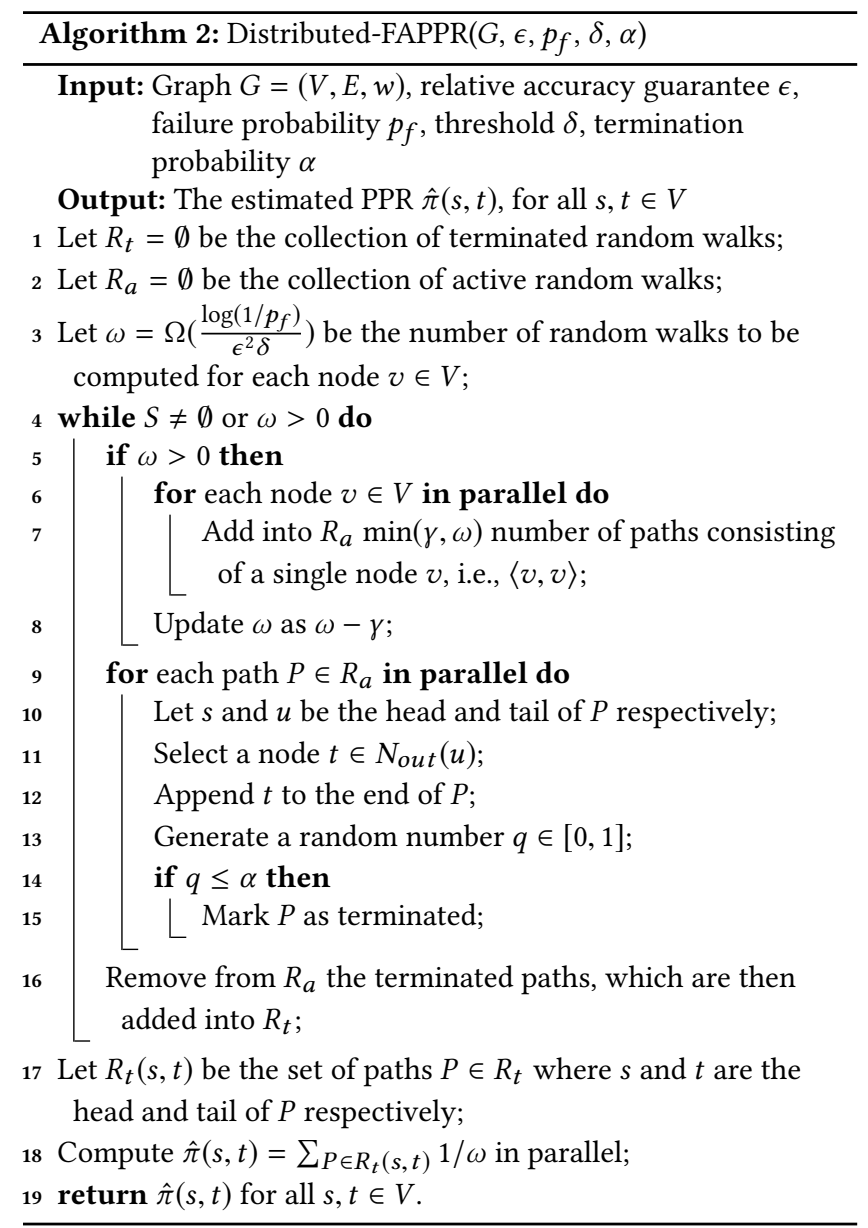

of paths for each node are computed simultaneously. However, this approach would incur significant overhead in the management of memory, as $\omega$ could be sufficiently large.

To remedy the aforementioned issues, we exploit the parallel pipeline framework, as depicted in Figure 2, where (i) each pipeline generates at most $\gamma \in[1, \omega]$ random walks for each node $v \in V$, and (ii) the pipelines begin sequentially, such that one pipeline $L$ starts only when the pipeline $L^{\prime}$, prior to $L$, completes one round of computation, i.e., the paths in $L^{\prime}$ are extended with one step. Therefore, there are $[\omega / \gamma\rceil$ pipelines, and the expected number of rounds in each pipeline is $1 / \alpha$. That is, the parallel pipeline framework is expected to employ $(\lceil\omega / \gamma\rceil+1 / \alpha-1)$ number of rounds of computation, which is much smaller than the one of previous approaches. Besides, as each random walk could be terminated with the probability $\alpha$, the workload of each pipeline becomes fewer and fewer as the number of iterations increases. Therefore, we can avoid the under-utilization of computing for long pipelines, and make sure that the pipeline $L$ would receive few affections from the pipelines that are far from $L$.

Algorithm 2 depicts an overview of the proposed algorithm that takes as input the graph $G=(V, E, w)$, the relative accuracy guarantee $\epsilon$, failure probability $p_{f}$, threshold $\delta$, and termination probability $\alpha$. Initially, we have the empty collections of terminated 


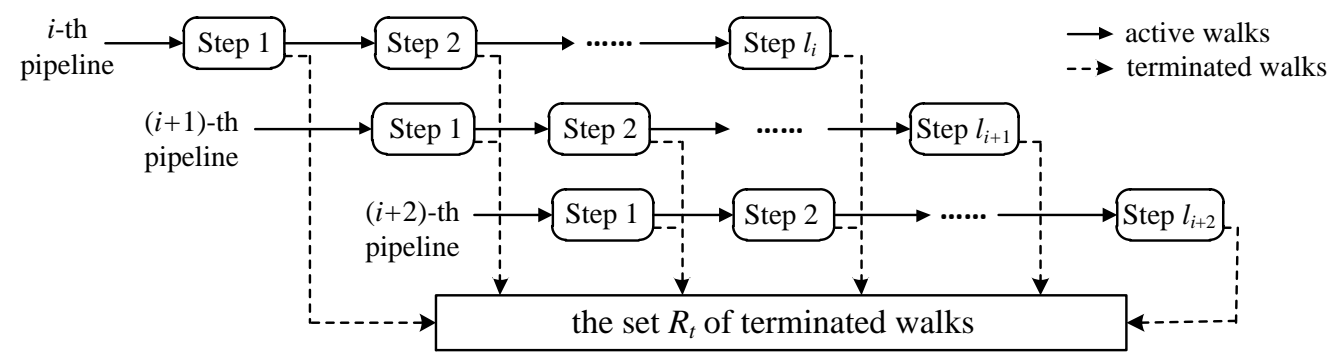

Figure 2: This is an illustration of the parallel pipeline framework. In this framework, there are $\lceil\omega / \gamma\rceil$ pipelines, each of which generates at most $\gamma$ random walks by several steps. For each $i \in[1,\lceil\omega / \gamma\rceil)$, the $(i+1)$-th pipeline starts, only when the random walks in the $i$-th pipeline have completed the first step. All terminated walks in the pipelines are maintained in the set $R_{t}$, which are the final output of the computation of random walks.

and active random walks, referred to as $R_{t}$ and $R_{a}$ respectively (Lines 1-2). Besides, the number $\omega$ of random walks is calculated as $\Omega\left(\frac{\log \left(1 / p_{f}\right)}{\epsilon^{2} \delta}\right)$. Then, we generate the random walks by several iterations (Lines 4-16). Each iteration consists of three phases, as follows. In the first phase, for each node $v \in V$, if $\omega>0$, then we create $\min (\gamma, \omega)$ number of paths $\langle v, v\rangle$ consisting of a single node $v$, and decrease $\omega$ by $\gamma$ (Lines 5-8). In the second phase, for each path $P \in R_{a}$, we extend $P$ with one step by selecting a node $t$ the set of out-neighbors of the tail $u$ of $P$ according to the routing probability $r(u, t)$. After that, we append $t$ to the end of $P$ and mark $P$ as terminated if $\alpha$ is not smaller than a random number generated on the fly (Lines 12-15). Finally, we remove from $R_{a}$ the terminated paths, which are then added into $R_{t}$ (Line 16). At the end of the algorithm, to compute $\hat{\pi}(s, t)$ for all $s, t \in V$, we calculate the number of paths $P$ in $R_{t}$ such that the head and tail of $P$ are $s$ and $t$ respectively.

\section{OPTIMIZATION TECHNIQUES}

In this section, we develop several efficient techniques that significantly improve the performance of Algorithm 2.

\subsection{Handling Large Nodes: The Alias Tree}

As explained previously, we adopt the alias method which allows us to select one node $t$ from $s$ 's out-neighbors, where $s \in V$, according to the routing probability with constant time and linear space complexity. However, when the degree of a node $u \in V$ is sufficiently large, due to that the size of $N_{\text {out }}(u)$ could be too large to fit in memory. To avoid this deficiency, we devise a hierarchical approach that recursively divides $N_{\text {out }}(u)$ into several subsets, each of which has a size not bigger than $d$, that can fit in memory, where $d$ is a user-defined parameter and can be estimated according to the amount of available memory. Then, we denote each subset $S^{\prime} \subseteq S$ as a new item with a weight equal to $\sum_{e \in S^{\prime}} r(e)$. As such, we construct a new set $\mathcal{S}$ of items, whose alias and switch probability can be computed by the alias method on subsets of $S$. We build the alias tree by letting the alias and switch probability of $S^{\prime}$ be a child of $\mathcal{S}$ for each item $S^{\prime} \in \mathcal{S}$. Apparently, the alias tree is multi-way tree, where each node has a similar number of children. If the size of the set $\mathcal{S}$ of the subsets exceeds the size of memory, we recursively divide $\mathcal{S}$ in the same way.

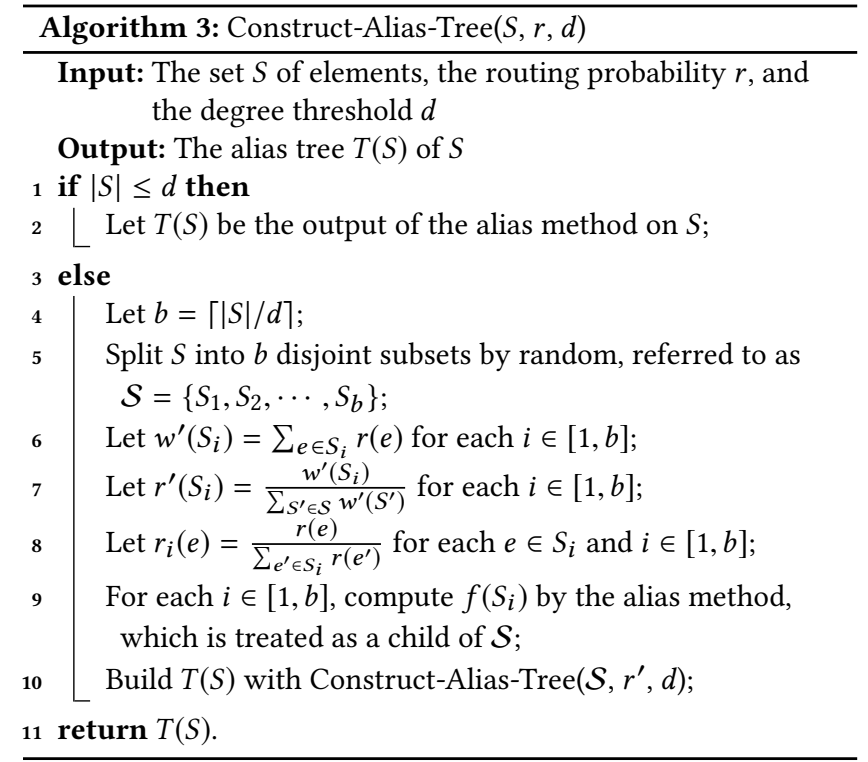

Algorithm 3 provides the pseudo-code of the construction of an alias tree on a given set $S$ of items. The algorithm first inspects the size of $S$. If its size is not bigger than $d$, then the algorithm returns the alias directly computed on $S$ by the alias method, which forms a single node of the alias tree (Line 2). Otherwise, we randomly split $S$ into $b$ disjoint subsets (Line 5). Denote the set of subsets by $\mathcal{S}=$ $\left\{S_{1}, S_{2}, \cdots, S_{b}\right\}$. Hence, we have $\cup_{S^{\prime} \in \mathcal{S}} S^{\prime}=S$ and $\cap_{S^{\prime} \in \mathcal{S}} S^{\prime}=\emptyset$. Then, we compute the weight of $S^{\prime} \in \mathcal{S}$, which is the sum of routing probability of items in $S^{\prime}$, referred to as $w^{\prime}\left(S^{\prime}\right)=\sum_{e \in S^{\prime}} r(e)$. Then, for each subset $S_{i}$ where $1 \leq i \leq b$, the routing probability of $S_{i}$ with respect to $\mathcal{S}$ is computed as $r^{\prime}\left(S_{i}\right)=\frac{w^{\prime}\left(S_{i}\right)}{\sum_{S^{\prime} \in \mathcal{S}} w^{\prime}\left(S^{\prime}\right)}$ (Line 7). Besides, to compute the alias of each item $e$ in each subset $S_{i}$, the routing probability of $e$ with respect to $S_{i}$ is updated as $r^{\prime}\left(S_{i}\right)=\frac{w^{\prime}\left(S_{i}\right)}{\sum_{S^{\prime} \in \mathcal{S}} w^{\prime}\left(S^{\prime}\right)}$ (Line 8). As a result, the child $f\left(S_{i}\right)$ of $\mathcal{S}$ is created by performing the alias method on the updated routing probability of items in $S_{i}$ (Line 9). Finally, we recursively construct the alias tree on $\mathcal{S}$, until the size of $\mathcal{S}$ fits in memory, i.e., not bigger than $d$ (Line 10). 


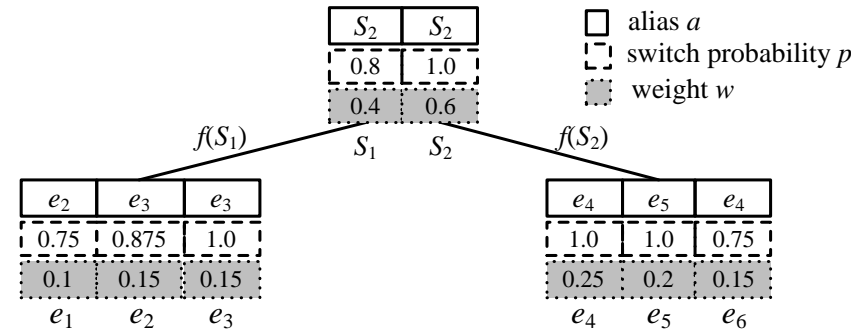

Figure 3: An example of the alias tree.

Example 4.1. Consider the 6 out-going edges of $v_{0}$ in Figure 1, denoted by $e_{1}, e_{2}, \cdots, e_{6}$ respectively. As shown, in Figure 3, the alias tree stores the 6 edges by treating them as 6 items in a set $S$. Assume that the degree threshold is $d=3$. As the size of $S$ is larger than $d$, we randomly divide $S$ into two sets, e.g., $S_{1}=\left\{e_{1}, e_{2}, e_{3}\right\}$ and $S_{1}=\left\{e_{4}, e_{5}, e_{6}\right\}$. Hence, we have $w^{\prime}\left(S_{1}\right)=r\left(e_{1}\right)+r\left(e_{2}\right)+r\left(e_{3}\right)=0.4$. Similarly, we have $w^{\prime}\left(S_{2}\right)=0.6$. As such, to obtain the routing probabilities, we can normalize the weights of elements in $S_{1}, S_{2}$, and $S$ respectively. Then, by utilizing the alias method, we construct the aliases and switch probabilities of $S_{1}$ and $S_{2}$, which form the children of $\mathcal{S}$. After that, we recursively construct the alias of $\mathcal{S}$, which is returned as shown in Figure 3, since the size of $\mathcal{S}$ is less than 3 .

To select an item, we traverse the alias tree $T(S)$ recursively by starting from the root $T$ of $T(S)$. To explain, we first select an item $e$ from $T$ with a probability $1 /|T|$, which can be achieved in $O(1)$ time. Then, a random number $r \in[0,1]$ is generated. If $r \leq p(e)$, then we select $e$, otherwise $a(e)$. Afterwards, if $f(e)$ is not empty, i.e., $e$ has children, we recursively inspect the items in $f(e)$; otherwise, we return $e$ as the result. Apparently, The running time of the selection process is determined by the height of the alias tree, i.e., $O(\log (|S|) / \log (d))$, where $d$ is the degree threshold. Lemma 1 provides the correctness of random item generation based on the alias tree.

Example 4.2. Consider the alias tree in Example 2.3. To select an element, it consists of two steps: First, we select an element $S^{\prime}$ from $\mathcal{S}$; Then, an element $e$ is selected from $S^{\prime}$. In the first step, assume that $S_{1}$ is selected with the probability $1 /|\mathcal{S}|=0.5$. After that, we generate a random number $r \in[0,1]$. If $r \leq p\left(S_{1}\right)$, we select $S_{1}$, otherwise select $a\left(S_{1}\right)=S_{2}$. As a result, the probability of selecting $S_{1}$ is $0.5 \cdot p\left(S_{1}\right)=0.4$, which equals the normalized weight of $S_{1}$, i.e., the routing probability of $S_{1}$. Assume that $S_{1}$ is selected in the first step. We recursively select an element from $S_{1}$ in the same way, which leads to the final selection.

Lemma 1. Given a set $S$ of edges, as well as the alias tree $T$ constructed on $S$, the selection an elemente $\in S$ from $T$ has the probability equal to $r(e)$.

Proof. Let $\left\langle S_{1}, S_{2}, \cdots, S_{i}\right\rangle$ be the path from the root of $T$ to a leaf of $T$, where $e$ resides. Consider a node $S_{j}$, where $1 \leq j \leq i$. As shown previously, each item $x$ in $S_{j}$ is selected with the probability $w(x) / \sum_{y \in S_{j}} w(y)$. Besides, the weight of $S_{j}$ is the sum of weights of items in $S_{j}$, i.e., $w\left(S_{j}\right)=\sum_{y \in S_{j}} w(y)$. Note that,

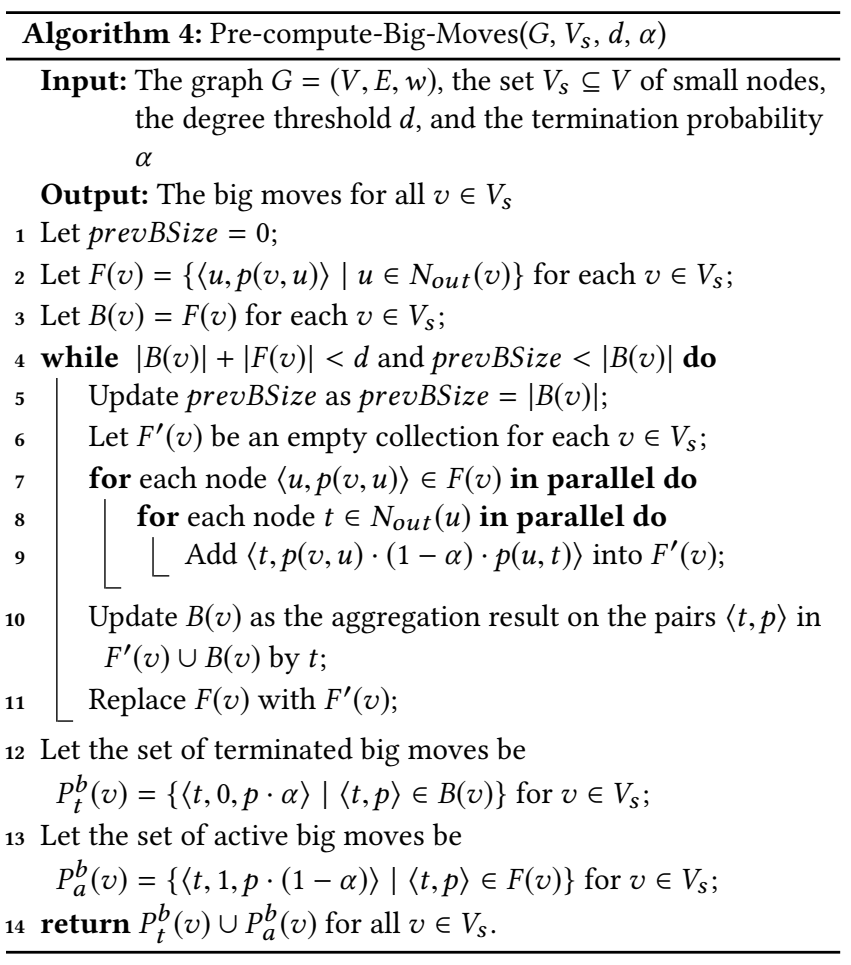

$w\left(S_{1}\right)=\sum_{e \in S} r(e)=1$, since $S_{1}$ represents all items in $S$. Therefore, the probability of selecting $e$ is

$$
\frac{w\left(S_{2}\right)}{w\left(S_{1}\right)} \cdot \frac{w\left(S_{3}\right)}{w\left(S_{2}\right)} \cdots \frac{w(e)}{w\left(S_{i}\right)}=\frac{r(e)}{w\left(S_{1}\right)}=r(e) .
$$

\subsection{Handling Small Nodes: Make Big Moves}

Most real-life graphs follow the power law [6], whose implication is that the majority of nodes in the graph have a very small set of neighbors (see Table 2). To speed up the random walks among the small-degree nodes, we devise a pre-computing method that generates the big moves for each small-degree node, such that each big move represents a random walk of several steps. To facilitate the computation with big moves, we maintain each big move $P^{b}$ with the similar structure as the normal random walk with a difference in that we add a mark, denoted by $M\left(P^{b}\right)$, to represent whether $P^{b}$ is terminated or not. If $M\left(P^{b}\right)=0$, then $P^{b}$ is a terminated path; otherwise, if $M\left(P^{b}\right)=1$, then $P^{b}$ is an active path. As a result, we can denote a big move of $v$ by $\left\langle u, t_{m}, p\right\rangle$ which are the walks ending at $u$ with the termination mark $t_{m}$ and the probability $p$. In other words, compared with the set of out-neighbors, the big moves consists of the event of termination, which expands the probability space.

Algorithm 4 describes the generation of big moves for each node $v$ in the set $V_{s}$ of small nodes. Initially, for each $v \in V_{s}$, we have the set $F(v)$ of active walks be the set of out-neighbors $u$ of $v$ with the accumulated probability equal to $p(v, u)$, i.e., $F(v)=\{\langle u, p(v, u)\rangle \mid$ $\left.u \in N_{\text {out }}(v)\right\}$. Besides, we let the set $B(v)$ of the terminated walks be initialized as $F(v)$, and we denote the size of $B(v)$ before updating 
(Line 10) by prevBSize, which is initialized as 0 . Note that, the initial sizes of $F(v)$ and $B(v)$ equal the size of $N_{\text {out }}(v)$. In what follows, the algorithm runs in several iterations. In each iteration, we extend each walk in $F(v)$ with one more step by adding each node $u \in N_{\text {out }}(v)$, resulting in a set $F^{\prime}(v)$ of new walks. Then, we compute the accumulated paths in both $B(v)$ and $F^{\prime}(v)$, and also update $F(v)$ with new walks (Lines 5-11). These processes are repeated in iterations, until (i) the number of terminated and active walks exceeds $d$, i.e., $|B(v)|+|F(v)| \geq d$, or (ii) the walks can not reach any new nodes, i.e., the size of $B(v)$ does not change any more, namely prevBSize $=|B(v)|$. Finally, we integrate the walks in $B(v)$ and $F(v)$ by multiplying the probability in $B(v)$ and $F(v)$ with $\alpha$ and $1-\alpha$ respectively, and adding the termination mark 0 and 1 respectively, which leads to resulting set $B M(v)$ of big moves of $v$.

Example 4.3. Consider the graph $G=(V, E, w)$ in Figure 1. Assume that the degree threshold $d=4$ and the termination probability $\alpha=0.5$. Then, all the nodes $v \in V$, except $v_{0}$, need to compute their big moves, since $\left|N_{\text {out }}(v)\right|<d$. Consider node $v_{2}$, which has one out-neighbor $v_{3}$. To compute the big move of $v_{2}$, we first initialize the set $F\left(v_{2}\right)$ as $\left\{\left\langle v_{3}, 1\right\rangle\right\}$ and the set $B\left(v_{2}\right)=F\left(v_{2}\right)$. Since $\left|F\left(v_{2}\right)\right|+\left|B\left(v_{2}\right)\right|=2<d$, we extend each walk in $F\left(v_{2}\right)$ by one step, and obtain the set $F^{\prime}\left(v_{2}\right)=\left\{\left\langle v_{4}, 1 \times 0.5 \times 1=0.5\right\rangle\right\}$ as $v_{3}$ has only one neighbor, i.e., $v_{4}$. Then, we update prevBSize $=\left|B\left(v_{2}\right)\right|=1$, and update $B\left(v_{2}\right)$ as the aggregation of items in $B\left(v_{2}\right)$ and $F^{\prime}\left(v_{2}\right)$, resulting in the set $\left\{\left\langle v_{3}, 1\right\rangle,\left\langle v_{4}, 0.5\right\rangle\right\}$. In addition, $F\left(v_{2}\right)$ is updated as $F^{\prime}\left(v_{2}\right)$, i.e., $F\left(v_{2}\right)=\left\{\left\langle v_{4}, 0.5\right\rangle\right\}$. Afterwards, we continue to extend the walks in $F\left(v_{2}\right)$, since $\left|F\left(v_{2}\right)\right|+\left|B\left(v_{2}\right)\right|=3<d$, and we have $F^{\prime}\left(v_{2}\right)=\left\{\left\langle v_{3}, 0.5 \times 0.5 \times 1=0.25\right\rangle\right\}$. Now, we update prevBSize $=\left|B\left(v_{2}\right)\right|=2$, update $F\left(v_{2}\right)=F^{\prime}\left(v_{2}\right)$, and update $B\left(v_{2}\right)$ as the aggregation of items in $F^{\prime}\left(v_{2}\right)$ and $B\left(v_{2}\right)$, leading to the set $\left\{\left\langle v_{3}, 1.25\right\rangle,\left\langle v_{4}, 0.5\right\rangle\right\}$. Because prevBSize $=\left|B\left(v_{2}\right)\right|$, i.e., the size of $B\left(v_{2}\right)$ does not change any more, we stop the iteration, and compute the set $P_{t}^{b}\left(v_{2}\right)$ of terminated big moves as $\left\{\left\langle v_{3}, 0,1.25 \times 0.5=0.625\right\rangle,\left\langle v_{4}, 0,0.5 \times 0.5=0.25\right\rangle\right\}$ and the set $P_{a}^{b}\left(v_{2}\right)$ of active big moves as $\left\{\left\langle v_{3}, 1,0.25 \times 0.5=0.125\right\rangle\right\}$. As a result, we have the big moves of $v_{2}$ as the set $B M\left(v_{2}\right)=$ $P_{t}^{b}\left(v_{2}\right) \cup P_{a}^{b}\left(v_{2}\right)=\left\{\left\langle v_{3}, 0,0.625\right\rangle,\left\langle v_{4}, 0,0.25\right\rangle,\left\langle v_{3}, 1,0.125\right\rangle\right\}$.

We show the correctness of Algorithm 4 by Lemma 2.

LEMMA 2. Given a set BM(v) of big moves of $v$, then $B M(v)$ consists of all random walks starting from $v$ within $k$ steps, where $k$ is the longest length of walks in $B M(v)$.

Proof. It is sufficient to show that (i) if there exists a path of length at most $k$ that starts from $v$ and ends at $u$, then $u$ must be in $B M(v)$; and (ii) $\sum\left\langle v, t_{m}, p\right\rangle \in B M(v) p=1$.

In the first case, we are to show that all paths, whose lengths are not larger than $k$, are in $B M(v)$. Let $P\left(v, u_{t}\right)=\left\langle v, u_{1}, u_{2}, \cdots, u_{t}\right\rangle$ be a path of length $t \leq k$ that starting from $v$. In the first round, we have $u_{1}$ selected, as $u_{1}$ is an out-neighbor of $v$, i.e., $u_{1} \in N_{\text {out }}(v)$. By induction, $u_{i+1}$ is an out-neighbor of $u_{i}$, where $1 \leq i<t$. As such, the sequence $\left\langle v, u_{1}, u_{2}, \cdots, u_{t}\right\rangle$ is a path of $G$.

In the second case, we need to demonstrate that the probability space of $B M(v)$ is completed. Let $p_{t}$ be the probability that a random walk $P=\left\langle v, u_{1}, u_{2}, \cdots u_{t}\right\rangle$ is of length $t$. Hence, we have

$$
\begin{aligned}
p_{t} & =\sum_{\ell(P)=t} p\left(\left\langle v, u_{1}\right\rangle\right) p\left(\left\langle u_{1}, u_{2}\right\rangle\right) \cdots p\left(\left\langle u_{t-1}, u_{t}\right\rangle\right)(1-\alpha)^{t-1} \\
& =(1-\alpha)^{t-1} .
\end{aligned}
$$

Consider the probability $p^{\prime}$ of the event that a random walk $P \in$ $B M(v)$ is a terminated path, which means that $P$ is of length $t \leq k$. As $B M(v)$ contains all paths of length not bigger than $k$, we have

$$
p^{\prime}=\alpha \cdot \sum_{1 \leq t \leq k} p_{t}=\alpha \cdot \sum_{1 \leq t \leq k}(1-\alpha)^{t-1}=1-(1-\alpha)^{k} .
$$

Besides, consider the probability $p^{\prime \prime}$ of the event that a random walk $P \in B M(v)$ is an active path, which indicates that $P$ is of length $k$. Therefore, we have $p^{\prime \prime}=(1-\alpha) \cdot p_{k}=(1-\alpha)^{k}$. To sum up, we obtain $\sum_{\left\langle v, t_{m}, p\right\rangle \in B M(v)} p=p^{\prime}+p^{\prime \prime}=1$, which completes the proof.

To continue the generation of the random walk ending with the node $v$, which is a small node with the set $B M(v)$ of big moves, we randomly select a path $P^{b}=\left\langle v, t_{m}, p\right\rangle \in B M(v)$ following the distribution of probabilities of paths in $B M(v)$. Note that, the selection can be accomplished in constant time with the alias method. If $t_{m}=0$, then $P^{b}$ is a terminated path, and we add $P^{b}$ into the set $R_{t}$. Otherwise, we continue the walk along the path $P^{b}$ until the termination.

\subsection{Optimizing $\gamma$}

Our previous discussion has focused on the generation of random walks in the graph $G$. When the number $\omega$ of random walks is considerably large, it could be highly inefficient to generate all random walks for all nodes of $G$ at a time, as discussed in the previous sections. To remedy this deficiency, we proposed the parallel pipeline framework that processes the $\omega$ number of random walks by several iterations, each of which generates at most $\gamma$ random walks for each node of $G$. That is, there will be $\lceil\omega / \gamma\rceil$ number of pipelines.

One crucial question remains: how do we decide the number $\gamma$ of random walks in each pipeline? A straightforward approach is to set $\gamma=1$, i.e., we generate one random walk for each node $v \in V$ in each pipeline. Although this can avoid the memory issues as aforementioned, it brings up the other deficiencies where (i) the probability to having a long walk is low, which leads to the underutilization of computing resources, and (ii) the number of pipelines would be a lot, which increases the running time. To tackle these issues, we devise a heuristic method to choose $\gamma$, as follows. First, in our implementation, we maintain a path $P$ by keeping the head $s$ and the tail $t$ of $P$, i.e., $\langle s, t\rangle$. As such, it is a constant space cost $c$ for a path $P$. Second, in the parallel pipeline framework, we start the pipelines sequentially such that one latter pipeline $L$ is started only upon that the pipeline $L^{\prime}$, prior to $L$, completes the extension of random walks in $L^{\prime}$ by one step. Specifically, let $s_{i}$ be the total number of active random walks that are generated by the first $i$ pipelines. After the extension of one step at the $i$-th round, the number of remaining random walks is expected to be $(1-\alpha) \cdot s_{i}$. In the $(i+1)$-th pipeline, we start another $n \cdot \gamma$ number of new random walks in total, where $n$ is the number of nodes of $G$. To sum up, the total expected number of random walks to be processed at 
Table 2: Datasets.

\begin{tabular}{|c|r|r|r|r|r|r|}
\hline Name & $|V|$ & $|E|$ & $d_{a v g}$ & $d_{\max }$ & $\left|V_{s}\right| /|V|$ & $\left|V_{l}\right| /|V|$ \\
\hline GrQc & 5,241 & 28,968 & 5.5 & 81 & $78.3 \%$ & $15.9 \%$ \\
\hline CondMat & 23,133 & 186,878 & 8.1 & 279 & $75.4 \%$ & $12.2 \%$ \\
\hline Enron & 36,692 & 367,662 & 10.0 & 1383 & $85.5 \%$ & $5.1 \%$ \\
\hline DBLP & 317,080 & $1,049,866$ & 5.6 & 306 & $46.9 \%$ & $4.2 \%$ \\
\hline Stanford & 281,903 & $2,312,497$ & 8.2 & 255 & $75.5 \%$ & $14.7 \%$ \\
\hline Google & 875,713 & $5,105,039$ & 6.9 & 456 & $53.6 \%$ & $1.9 \%$ \\
\hline Skitter & $1,696,415$ & $11,095,298$ & 11.5 & 35,387 & $51.0 \%$ & $0.3 \%$ \\
\hline Patents & $3,774,768$ & $16,518,947$ & 7.9 & 770 & $38.5 \%$ & $1.5 \%$ \\
\hline Pokec & $1,632,803$ & $30,622,564$ & 21.4 & 8,763 & $61.0 \%$ & $2.6 \%$ \\
\hline Livefournal & $4,846,609$ & $68,475,391$ & 15.9 & 20,292 & $66.5 \%$ & $1.0 \%$ \\
\hline Orkut & $3,072,441$ & $117,185,083$ & 43.0 & 33,007 & $65.7 \%$ & $3.3 \%$ \\
\hline Twitter & $41,652,230$ & $1,468,364,884$ & 36.6 & $2,997,469$ & $87.9 \%$ & $0.2 \%$ \\
\hline Friendster & $68,349,466$ & $2,586,147,869$ & 46.1 & 5,214 & $65.5 \%$ & $12.8 \%$ \\
\hline
\end{tabular}

the $(i+1)$-th round is $s_{i+1}=n \cdot \gamma+(1-\alpha) \cdot s_{i}$. Note that, at the beginning, we have $s_{1}=n \cdot \gamma$. By solving these formulas, we have

$$
s_{i}=n \cdot \gamma \cdot \sum_{1 \leq j \leq i}(1-\alpha)^{j-1}=n \cdot \gamma \cdot \frac{1-(1-\alpha)^{i}}{\alpha}<\frac{n \cdot \gamma}{\alpha} .
$$

As such, if the size of available memory on a machine is $M$, then we have $c \cdot n \cdot \gamma / \alpha \leq M$. That is, we obtain $\gamma \leq \alpha \cdot M /(n \cdot c)$. Therefore, we can set $\gamma$ to the maximum integer such that the total size of processed random walks does not exceed the size of available memory, which helps the full utilization of the cluster. By experiments as shown later, we demonstrate that our choice of $\gamma$ results in good performance of the proposed algorithm.

\section{EXPERIMENTS}

This section presents the thorough experimental studies on the performance of the proposed algorithm, compared with the stateof-the-art approach, on several real-life datasets.

\subsection{Experimental Settings}

We implement our distributed algorithm for the computation of FAPPR (dubbed as DistPPR) in Scala on Spark 2.0 ${ }^{1}$, and compare it against two state-of-the-art distributed algorithms: Doubling [2] and the PPR library in GraphX ${ }^{2}$ (denoted as GXPPR). Note that, (i) Doubling is a MapReduce algorithm, which can be easily implemented on Spark, and (ii) GXPPR is a matrix-based method that iteratively computes with the adjacency matrix of the graph until the convergence of PPR. All of our experiments are conducted on an in-house cluster consisting of up to 201 machines, each of which runs CentOS, and has 16GB memory and 12 Intel Xeon Processor E5-2670 CPU cores. By default, we exploit 51 machines to run the algorithms, where we have one machine as the master and the others as the workers in Spark.

We use 13 real-life networks in our experiments, as shown in Table 2. These data are obtained from the SNAP collection ${ }^{3}$, and are intensively evaluated in the literatures [27, 28]. Besides, these data are from various categories: $G r Q c$ and CondMat are collaboration networks; Enron is a communication network; Standford and Google

\footnotetext{
${ }^{1}$ https://spark.apache.org/

${ }^{2}$ https://spark.apache.org/graphx/

${ }^{3}$ http://snap.stanford.edu/data/index.html
}

are the web graphs; Skitter is a internet topology network; Patents and DBLP are the citation networks; Pokec, Livefournal, Orkut, Twitter, and Friendster are social networks. Furthermore, these data have different sizes, ranged from thousands of edges to billions of edges. Their average out-degrees (denoted by $d_{a v g}$ ) are relatively small, though their maximum out-degrees (denoted by $d_{\text {max }}$ ) could be significantly large that are more than 2 million. Let $V_{s}$ be the set of small nodes whose out-degrees are less than $d_{a v g}$, and let $V_{l}$ be the set of large nodes whose out-degrees are more than $\sqrt{d_{\max }}$. As shown in Table 2, the small nodes are the majorities in the graph, while the large nodes take only a small portion. Following previous work [29], we adopt the linear parameterization to assign each edge a positive weight for each graph.

In addition, according to previous work [7,27], we set the failure probability $p_{f}$ to $1 / n$ where $n$ is the number of nodes in the graph, and set the default value of the relative accuracy guarantee $\epsilon$, the threshold $\delta$, and the termination probability $\alpha$ to 0.5 . Besides, if the algorithm does not terminate within 24 hours, we omit it from the experiments. We repeat each experiment 3 times and report the average reading of each approach.

\subsection{Comparisons with Previous Work}

In the first set of experiments, we demonstrate the superiority of our proposed algorithm by evaluating the running time of all the algorithms on all datasets. Note that, the running time of DistPPR includes both the time of the optimization techniques, i.e., we compare the running time of all algorithms with the same input data. Figure 5(a) shows the results of DistPPR, Doubling, and GXPPR on the datasets in Table 2. DistPPR outperforms all the other methods by up to 2 orders of magnitude on all datasets. This is because DistPPR addresses the issues of both large and small nodes and is able to leverage the massive parallelism of the distributed computing. However, Doubling spends lots of efforts on combining the short walks that results in a huge combinational space, and GXPPR incurs significant overhead in the operations of matrix which could be highly sparse. Besides, GXPPR is able to process only two small datasets, which again demonstrates that the matrix-based methods are difficult to handle large graphs that require huge memory space. Furthermore, the results of Doubling on the datasets, Livefournal, Orkut, Twitter, and Friendster, are omitted, since the running time of Doubling on those datasets exceeds 24 hours. On the contrary, DistPPR is able to handle all datasets with a relatively short running time. What's more, compared to Doubling and GXPPR, the performance of DistPPR is less sensitive to the number of edges in the graph.

Besides, to evaluate the accuracy of the approximate solutions, we generate the ground-truth by the exact PPR algorithm [29] that is able to process the graphs with less than ten millions edges on a machine with $48 \mathrm{~GB}$ memory. Based on that, we evaluate the accuracy of DistPPR and Doubling on 6 datasets by NDCG and MAP, which are widely used to evaluate the results of ranking ${ }^{4}$. In particular, given the graph $G=(V, E, w)$, for each node $v \in V$, we rank the nodes $u \in V$ by the value $\pi(v, u)$. We take the first 1000 nodes from each ranking for evaluation. Figure 6 shows the results of NDCG and MAP on several datasets for both DistPPR

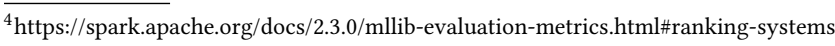




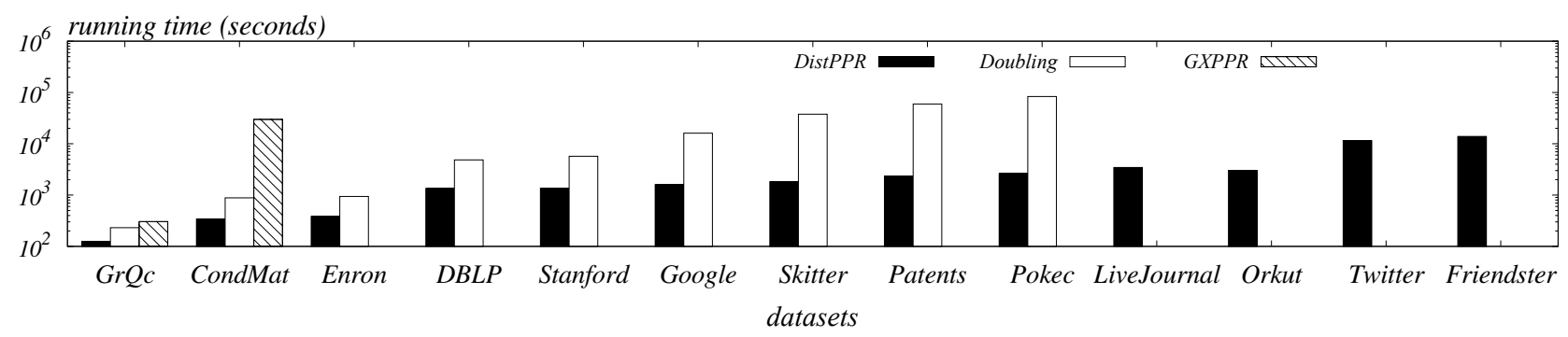

Figure 4: Running time on real-life datasets.

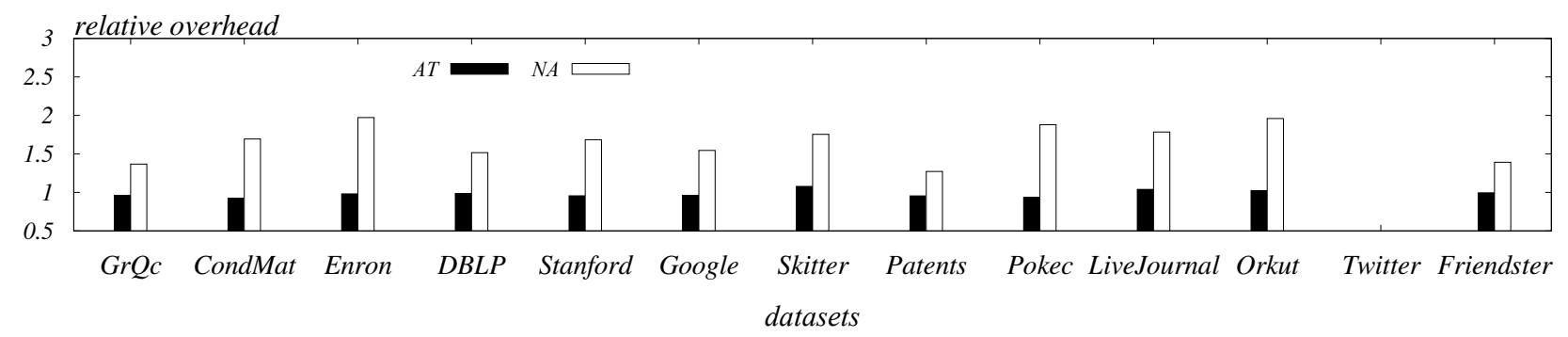

Figure 5: Effects of optimizations.

and Doubling respectively. On all datasets, the NDCG of DistPPR outperforms the one of Doubling by up to $21 \%$, and the MAP of DistPPR is also better than the one of Doubling by up to $19 \%$. This is because Doubling does not guarantee the accuracy of results, whereas DistPPR has a strong accuracy guarantee according to Definition 2.2.

\subsection{Effects of Optimizations}

In this section of experiments, we study the effects of the three proposed optimization techniques: the alias tree (AT) method for handling large nodes, the big move (BM) technique for accelerating the walks on small nodes, and the technique that auto-tunes $\gamma$.

We first evaluate the techniques of AT and BM. We consider two versions of algorithms modified from DistPPR: DistPPR but with the two optimizations disabled (denoted as NA), and DistPPR but with only the AT enabled (denoted as $A T$ ). We define the relative overhead of each modified version of DistPPR on a dataset $D$ as its running time of $D$ divided by the running time of DistPPR with all optimizations enabled. Figure 5(b) shows the relative overheads of $A T$ and NA on each dataset. Note that, the results on Twitter are not shown, as both $A T$ and NA fail to process Twitter whose node degree is highly skew. The relative overheads of $A T$ are around 1 in all cases, which indicates that the AT technique allows the algorithm avoiding the issues of large nodes and brings very few overheads in the running time. Meanwhile, the relative overhead of $N A$ is around 1.5 on all datasets, implying that the BM technique improves the efficiency of DistPPR by a factor of 1.5 .

Then, we evaluate the performance of the technique that automatically tunes $\gamma$. Recall that $\gamma \leq \omega$ is the parameter in the parallel pipeline framework that decides the number of random walks to generate in a pipeline. To evaluate our choice of $\gamma$, we measure the running time of DistPPR on the largest dataset Friendster by varying $\gamma$ from 30 to 480 , and compare with our autotune technique. We plot the results in Figure 7(a). Observe that the optimal value for $\gamma$ is around 120, and our automatic choice of $\gamma$ leads to a performance identical to the optimum. On the contrary, compared to the optimum, when $\gamma \leq 120$, it leads to longer running time, since the number of pipelines increases. Note that, the number of pipelines is $\lceil\omega / \gamma\rceil$. On the other hand, when $\gamma \geq 240$, it overloads the cluster with an excessive number of random walks to process simultaneously, which degrades the performance of DistPPR.

In summary, the three optimization techniques improve the efficiency of DistPPR by up to 2 times, and help scale DistPPR to large graphs whose node degrees could be highly skew.

\subsection{Varying Other Parameters}

In the last set of experiments, we study the sensitive of DistPPR to the other parameters, i.e., the number of workers on Spark and the termination probability $\alpha$. Note that, we omit the studies on the threshold $\delta$, as $\delta$ has the same effect as $\alpha$ to the number $\omega$ of random walks. We adopt the largest dataset Friendster in all subsequent experiments.

Figure 7(b) reports the running time of DistPPR on Friendster dataset by varying the number of workers on Spark. When we increase the number of workers, the running time of DistPPR decreases accordingly. However, when the number of workers ranges from 100 to 200, the decrease rate of DistPPR's running time becomes low, since the cost of communication between workers increases.

Figure 7(c) shows the performance of DistPPR by varying the termination probability $\alpha$. When $\alpha$ increases, it increases the probability of a random walk to be terminated at a certain step, leading to the decrease of the total amount of computation. 


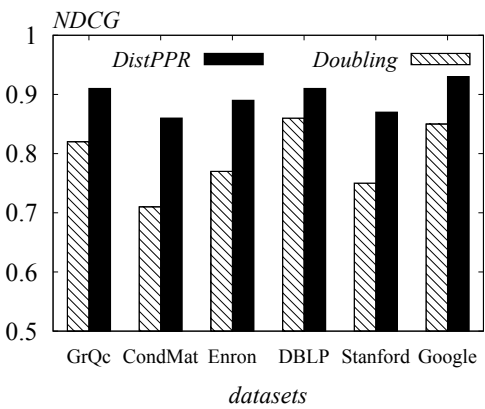

(a) NDCG.

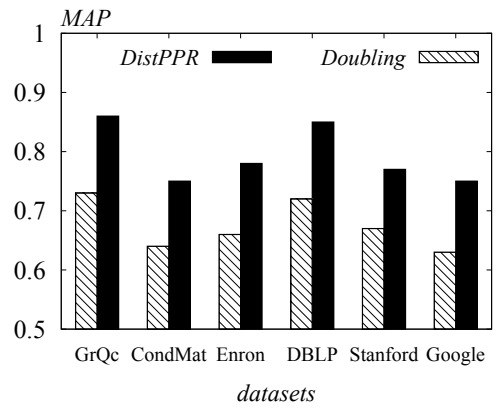

(b) MAP.

Figure 6: Accuracy comparison on NDCG and MAP.

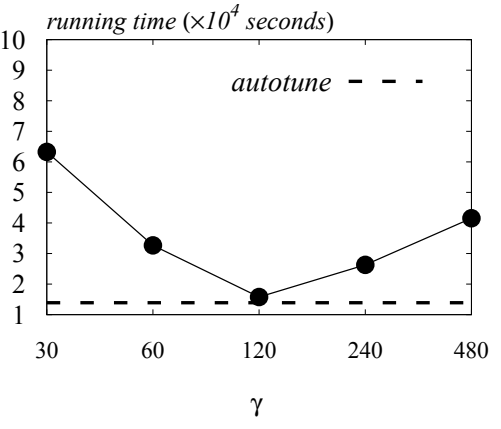

(a) Varying $\gamma$.

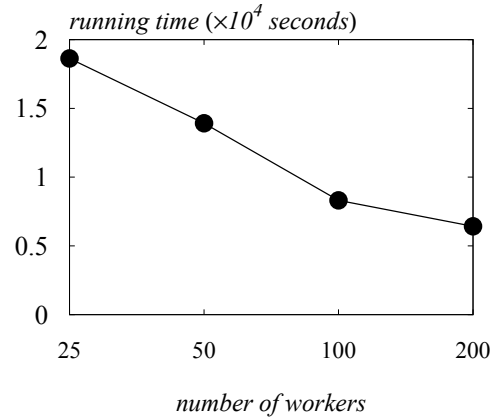

(b) Varying the number of workers.

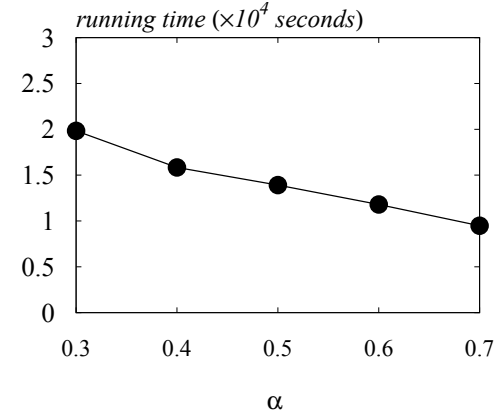

(c) Varying $\alpha$.

Figure 7: Varying parameters.

\section{RELATED WORK}

There exist some work [2, 22] that exploit the distributed computing techniques to accelerate the computation of the fully PPR or PR on large graphs based on the Monte Carlo approximation. They first generate some random walks of relatively short length, and then combine the short walks to obtain the random walks of long length. In particular, given two short walks $P_{1}=\left\langle v_{h 1} \cdots v_{t 1}\right\rangle$ and $P_{2}=\left\langle v_{h 2} \cdots v_{t 2}\right\rangle$, we can combine $P_{1}$ and $P_{2}$ if and only if the tail $v_{t 1}$ of $P_{1}$ equals the head $v_{h 2}$ of $P_{2}$, i.e., $v_{t 1}=v_{h 2}$, which results in a path $P=\left\langle v_{h 1}, \cdots, v_{t 1}, \cdots, v_{t 2}\right\rangle$ of length $\ell\left(P_{1}\right)+\ell\left(P_{2}\right)-1$. To make the combined paths sufficiently random, however, the number of short walks could be extremely large, especially for the large-degree nodes, rendering these approaches inefficient. Besides, there is no guarantee on the accuracy of the results produced by these approaches [2, 22].

Besides, some distributed algorithms are proposed to answer the queries of single-pair PPR [16] or single-source PPR [9, 11, 16], which differ from the problem of this paper that focuses on the computation of fully PPR. Additionally, there are some distributed and parallel algorithms for graph data $[10,14,15]$, but they do not aim for the problem of fully PPR computation.

On the other hand, Fogaras et al. [7] developed an approach on a single machine for the fully PPR by the Monte Carlo approximation. In particular, to facilitate the traversal on the graph, their approach relies on an indexing structure of the graph that is required by the computation of random walks for all nodes, which renders this approach difficult to be implemented as a distributed algorithm. Moreover, there also exist a plethora of techniques for processing the queries of single-pair PPR [17, 18, 26], single-source PPR [12, 24,27 ], and top- $k$ PPR [28] on a single machine, all of which have a different problem setting against the fully PPR. Besides, these techniques often require a preprocessing step, which are sequential algorithms, rendering them difficult to be translated in parallel.

Finally, Xie et al. [29] addressed an important issue that edgeweighted graphs widely exist in various applications, which is ignored by previous studies. However, their approach requires to compute based on the adjacency matrix of the graph, which could be prohibitively large.

\section{CONCLUSIONS}

This paper studies the problem of the fully Personalized PageRank, which has enormous applications in link prediction and recommendation systems for social networks. We approach this problem by devising an efficient distributed algorithm to accelerate the Monte Carlo approximation, which requires to generate a large number of random walks for each node of the graph. We exploit the parallel pipeline framework to achieve the superiority of cluster parallelism. To optimize the proposed algorithm, we develop three techniques that significantly improve the performance of the proposed algorithm in terms of efficiency and scalability. With extensive experiments on various real-life networks, we demonstrate that our proposed solution is up to 2 orders of magnitude faster than the start-of-the-art solutions, and also outperforms the baseline solutions on the evaluation of accuracy. 


\section{REFERENCES}

[1] Eneko Agirre and Aitor Soroa. 2009. Personalizing PageRank for Word Sense Disambiguation. In EACL 2009, 12th Conference of the European Chapter of the Association for Computational Linguistics, Proceedings of the Conference, Athens, Greece, March 30 - April 3, 2009. 33-41.

[2] Bahman Bahmani, Kaushik Chakrabarti, and Dong Xin. 2011. Fast personalized PageRank on MapReduce. In Proceedings of the ACM SIGMOD International Conference on Management of Data, SIGMOD 2011, Athens, Greece, fune 12-16, 2011. 973-984.

[3] Jeffrey Dean and Sanjay Ghemawat. 2004. MapReduce: Simplified Data Processing on Large Clusters. In 6th Symposium on Operating System Design and Implementation (OSDI 2004), San Francisco, California, USA, December 6-8, 2004 $137-150$.

[4] Pavlos S. Efraimidis and Paul G. Spirakis. 2006. Weighted random sampling with a reservoir. Inf. Process. Lett. 97, 5 (2006), 181-185.

[5] Chantat Eksombatchai, Pranav Jindal, Jerry Zitao Liu, Yuchen Liu, Rahul Sharma, Charles Sugnet, Mark Ulrich, and Jure Leskovec. 2018. Pixie: A System for Recommending 3+ Billion Items to 200+ Million Users in Real-Time. In Proceedings of the 2018 World Wide Web Conference on World Wide Web, WWW 2018, Lyon, France, April 23-27, 2018. 1775-1784.

[6] Michalis Faloutsos, Petros Faloutsos, and Christos Faloutsos. 1999. On Power-law Relationships of the Internet Topology. In SIGCOMM. 251-262.

[7] Dániel Fogaras, Balázs Rácz, Károly Csalogány, and Tamás Sarlós. 2005. Towards Scaling Fully Personalized PageRank: Algorithms, Lower Bounds, and Experiments. Internet Mathematics 2, 3 (2005), 333-358.

[8] Yasuhiro Fujiwara, Makoto Nakatsuji, Takeshi Yamamuro, Hiroaki Shiokawa, and Makoto Onizuka. 2012. Efficient personalized pagerank with accuracy assurance. In The 18th ACM SIGKDD International Conference on Knowledge Discovery and Data Mining, KDD '12, Beijing, China, August 12-16, 2012. 15-23.

[9] Tao Guo, Xin Cao, Gao Cong, Jiaheng Lu, and Xuemin Lin. 2017. Distributed Algorithms on Exact Personalized PageRank. In Proceedings of the 2017 ACM International Conference on Management of Data, SIGMOD Conference 2017, Chicago, IL, USA, May 14-19, 2017. 479-494.

[10] Qirong Ho, Wenqing Lin, Eran Shaham, Shonali Krishnaswamy, The Anh Dang, Jingxuan Wang, Isabel Choo Zhongyan, and Amy She-Nash. 2016. A Distributed Graph Algorithm for Discovering Unique Behavioral Groups from Large-Scale Telco Data. In Proceedings of the 25th ACM International Conference on Information and Knowledge Management, CIKM 2016, Indianapolis, IN, USA, October 24-28, 2016. 1353-1362.

[11] Jinhong Jung, Namyong Park, Lee Sael, and U. Kang. 2017. BePI: Fast and MemoryEfficient Method for Billion-Scale Random Walk with Restart. In Proceedings of the 2017 ACM International Conference on Management of Data, SIGMOD Conference 2017, Chicago, IL, USA, May 14-19, 2017. 789-804.

[12] Jung Hyun Kim, K. Selçuk Candan, and Maria Luisa Sapino. 2013. LR-PPR: localitysensitive, re-use promoting, approximate personalized pagerank computation. In 22nd ACM International Conference on Information and Knowledge Management, CIKM'13, San Francisco, CA, USA, October 27 - November 1, 2013. 1801-1806.

[13] Wei-keng Liao, Alok N. Choudhary, Donald D. Weiner, and Pramod K. Varshney 2005. Performance Evaluation of a Parallel Pipeline Computational Model for Space-Time Adaptive Processing. The Journal of Supercomputing 31, 2 (2005), 137-160.

[14] Wenqing Lin, Xiaokui Xiao, and Gabriel Ghinita. 2014. Large-scale frequent subgraph mining in MapReduce. In IEEE 30th International Conference on Dato Engineering, Chicago, ICDE 2014, IL, USA, March 31 - April 4, 2014. 844-855.

[15] Wenqing Lin, Xiaokui Xiao, Xing Xie, and Xiaoli Li. 2017. Network Motif Discovery: A GPU Approach. IEEE Trans. Knowl. Data Eng. 29, 3 (2017), 513-528.

[16] Qin Liu, Zhenguo Li, John C. S. Lui, and Jiefeng Cheng. 2016. PowerWalk: Scalable Personalized PageRank via Random Walks with Vertex-Centric Decomposition. In Proceedings of the 25th ACM International Conference on Information and
Knowledge Management, CIKM 2016, Indianapolis, IN, USA, October 24-28, 2016. 195-204.

[17] Peter Lofgren, Siddhartha Banerjee, and Ashish Goel. 2016. Personalized PageRank Estimation and Search: A Bidirectional Approach. In Proceedings of the Ninth ACM International Conference on Web Search and Data Mining, San Francisco, CA, USA, February 22-25, 2016. 163-172.

[18] Peter Lofgren, Siddhartha Banerjee, Ashish Goel, and Seshadhri Comandur. 2014. FAST-PPR: scaling personalized pagerank estimation for large graphs. In The 20th ACM SIGKDD International Conference on Knowledge Discovery and Data Mining, KDD '14, New York, NY, USA - August 24 - 27, 2014. 1436-1445.

[19] Siqiang Luo, Xiaokui Xiao, Wenqing Lin, and Ben Kao. 2019. Efficient Batch One-Hop Personalized PageRanks. In IEEE 35th International Conference on Data Engineering, Macau SAR, ICDE 2019, China, April 8 - April 12, 2019.

[20] Grzegorz Malewicz, Matthew H. Austern, Aart J. C. Bik, James C. Dehnert, Ilan Horn, Naty Leiser, and Grzegorz Czajkowski. 2010. Pregel: a system for large-scale graph processing. In Proceedings of the ACM SIGMOD International Conference on Management of Data, SIGMOD 2010, Indianapolis, Indiana, USA, fune 6-10, 2010. 135-146.

[21] Phuong Nguyen, Paolo Tomeo, Tommaso Di Noia, and Eugenio Di Sciascio. 2015. An evaluation of SimRank and Personalized PageRank to build a recommender system for the Web of Data. In Proceedings of the 24th International Conference on World Wide Web Companion, WWW 2015, Florence, Italy, May 18-22, 2015 Companion Volume. 1477-1482.

[22] Atish Das Sarma, Anisur Rahaman Molla, Gopal Pandurangan, and Eli Upfal. 2015. Fast distributed PageRank computation. Theor. Comput. Sci. 561 (2015), 113-121.

[23] Yufei Tao, Wenqing Lin, and Xiaokui Xiao. 2013. Minimal MapReduce algorithms. In Proceedings of the ACM SIGMOD International Conference on Management of Data, SIGMOD 2013, New York, NY, USA, June 22-27, 2013. 529-540.

[24] Hanghang Tong, Christos Faloutsos, and Jia-Yu Pan. 2006. Fast Random Walk with Restart and Its Applications. In Proceedings of the 6th IEEE International Conference on Data Mining (ICDM 2006), 18-22 December 2006, Hong Kong, China. 613-622.

[25] Michael D. Vose. 1991. A Linear Algorithm For Generating Random Numbers With a Given Distribution. IEEE Trans. Software Eng. 17, 9 (1991), 972-975.

[26] Sibo Wang, Youze Tang, Xiaokui Xiao, Yin Yang, and Zengxiang Li. 2016. HubPPR: Effective Indexing for Approximate Personalized PageRank. PVLDB 10, 3 (2016), 205-216.

[27] Sibo Wang, Renchi Yang, Xiaokui Xiao, Zhewei Wei, and Yin Yang. 2017. FORA: Simple and Effective Approximate Single-Source Personalized PageRank. In Proceedings of the 23rd ACM SIGKDD International Conference on Knowledge Discovery and Data Mining, Halifax, NS, Canada, August 13 - 17, 2017. 505-514.

[28] Zhewei Wei, Xiaodong He, Xiaokui Xiao, Sibo Wang, Shuo Shang, and Ji-Rong Wen. 2018. TopPPR: Top-k Personalized PageRank Queries with Precision Guarantees on Large Graphs. In Proceedings of the 2018 International Conference on Management of Data, SIGMOD Conference 2018, Houston, TX, USA, Fune 10-15, 2018. 441-456.

[29] Wenlei Xie, David Bindel, Alan J. Demers, and Johannes Gehrke. 2015. EdgeWeighted Personalized PageRank: Breaking A Decade-Old Performance Barrier. In Proceedings of the 21th ACM SIGKDD International Conference on Knowledge Discovery and Data Mining, Sydney, NSW, Australia, August 10-13, 2015. 13251334.

[30] Matei Zaharia, Mosharaf Chowdhury, Michael J. Franklin, Scott Shenker, and Ion Stoica. 2010. Spark: Cluster Computing with Working Sets. In 2nd USENIX Workshop on Hot Topics in Cloud Computing, HotCloud'10, Boston, MA, USA, fune 22,2010

[31] Wanxin Zhang, Dongsheng Li, Ying Xu, and Yiming Zhang. 2016. Shuffle-efficient distributed Locality Sensitive Hashing on spark. In IEEE Conference on Computer Communications Workshops, INFOCOM Workshops 2016, San Francisco, CA, USA, April 10-14, 2016. 766-767. 